\title{
VALORES EN EDUCACIÓN AMBIENTAL PARA LA PREVENCIÓN DE DESASTRES
}

\author{
Elsa Leonor Talero de Husain, Gloria Umaña*
} Gilma Chaves*

\begin{abstract}
The educational project on disaster prevention is an approximation towards a pedagogical strategy in significant learning, in values formation and concepts construction for the mountain system and the factors that affect the antropic steadystate equilibrium of the environment.
\end{abstract}

The design centers on teachers, students and community values clarification in order to incorporate geological hazards on vulnerability and risk of disasters. The main objective of the study was to design a curricula and to develop the educational. Project on disasters prevention that improve knowledge of existing hazard condition and distributions, as well as attitudes and values towards disasters.

The pedagogical .strategy tend, to minimize the risk of the disasters in the Andes mountains in which there are hazards such debris flows, rack-fall, subsidence and floods.

The values considered were: the person as center, the pertinence, property sense and self-steem. The curricula six students modules named "The people of the mountains'? The Mountains ecosystem, the livig beings, The Planet earth, and the machines and the Pedagogical project in disaster prevention for the teachers.

The curricula were validated la schools of Utica and Choachi (Cundinamarca department).

\section{PALABRAS CLAVES}

Vulnerabilidad amenaza, riesgo, prevención, valor, contravalor.

\section{INTRODUCCIÓN}

El proyecto educativo de prevención de desastres es una primera aproximación hacia un enfoque pedagógico, centrado en el aprendizaje significativo, en la formación de valores hacia la prevención y en la conceptuación sobre el sistema de montaña y los factores que alteran su equilibrio dinámico.

\footnotetext{
${ }_{\star \star}^{*}$ Docente Universidad Pedagógica Nacional.

${ }^{* *}$ Docente Universidad de Cundinamarca. 
El diseño responde a la necesidad de clarificar valores en los maestros, los educandos y la comunidad ante los problemas de amenaza, vulnerabilidad y riesgo en áreas altamente afectadas por desastres en zonas de lindero de la cordillera oriental, en el cual las áreas están expuestas a un alto riesgo de inundación, deslizamiento y avalancha.

\section{LOS VALORES EN LA PREVENCIÓN DE DESASTRES}

En la formación de valores, es necesario el aprendizaje para lograr una educación integral en el individuo. En formación interviene el entorno, los agentes socializadores y todo aquello que compone el contexto en el que se encuentra inmerso el ser humano.

Martínez (1994) afirma: se entiende por valores aquellos contenidos educativos que sirven de principio normativo que preside y regula el comportamiento de las personas en cualquier momento y situación".

Estos principios son los que regulan el comportamiento de los sujetos, pero su elección depende del contexto en donde se encuentre inmerso el individuo, del desarrollo cultural, histórico, económico, político, ambiental, religioso, artístico.

Existen varias escuelas que han dado explicaciones a los valores las cuales dependen de la justificación que le den a su doctrina: subjetiva, objetiva o sociológica.

“Para los subjetivistas, el 'agrado-desagrado' es el principio selector del valor por parte del individuo. Frente a ellos, la fenomenología de M. Scheller y N. Hartman (1970) considera los valores como ideas de vida constantes, a los que individuos y sociedad han de tender en su búsqueda de plenitud. Y el enfoque social añade el término conquista histórica para indicar que los valores surgen de la historia de los pueblos, de las experiencias y relaciones interpersonales, que llegan a formularse como principios categóricos y universales".

Es decir que los valores dependen del contexto histórico y cultural en donde esté inmerso el sujeto, de ahí que el niño retoma los valores de la observación que hace de los adultos, del contexto en donde se encuentra y de la cultura que tiene su comunidad.

Por otro lado, las diferentes interacciones con el profesor, le permite seguirlo, como el modelo que hay que imitar. Por eso es muy común que un niño de tres años que asiste a un jardín infantil, critique o no acepte alguna observación de sus padres, y les diga, eso no es así, porque mi profe" dijo que era de tal forma.

Así mismo, los niños imitan y se identifican con su profesor, especialmente los niños de preescolar y de primaria.

\section{La persona como centro}

Al ser humano se le debe considerar en su totalidad y unicidad, porque es único e irrepetible, posee unos rasgos y características muy especificas en su personalidad, estilo cognoscitivo, diferencias, aprendizajes, desarrollo motriz y afectividad.

En este momento en que la sociedad está pasando por una serie de progresos y desarrollo científico, técnico y de deterioro ambiental, es necesario preparar a los alumnos 
para que logre su realización pero no a costa de su propia destrucción, por lo tanto es necesario que el niño alcance los valores personales a través de la autonomía, la libertad y la acción. (Manrique, 1998)

'La autonomía como meta, como proceso, es decir, la formación de la conciencia capaz de establecer en sí misma el 'lugar de control', de ser el laboratorio donde se elaboren los criterios y juicios capaces de gobernar a la persona desde su propio dinamismo, sin someterse a los raques de alienación tan propios de la sociedad consumista trivializada".

Esta autonomía debe dirigir al niño a liderar procesos de mejoramiento personal buscando el desarrollo humano y social sin depender de las ordenes que el profesor le imparta, pero para ello el profesor debe llevar al niño a que no reflexione sobre todo el trabajo que realizan en el aula y los adultos deben compartir con él sus experiencias, lo deben hacer participe de sus decisiones, le deben colaborar y tratar de desarrollar en el la cooperación y la solidaridad.

Cuando se llega a una conciencia autónoma, se está alcanzando la madurez, el niño medita sus ideas, sus acciones y logra un buen juicio sobre lo que tiene que hacer o dejar de hacer.

La libertad, sin lugar a dudas, está íntimamente ligada a la autonomía y por tanto, como ella constituye un proceso. Ningún valor es tan ensalzado en nuestra sociedad como el de la libertad aunque a veces es confundida con 'las libertades', y ninguno penetra más profundamente en la conciencia de las personas como éste. Sin embargo, la educación ha de tener en cuenta los niveles de maduración de la libertad en su doble sentido: el de las manifestaciones de espontaneidad y el de la interiorización de los límites de la libertad por referencia al respeto a los otros".

Por lo tanto dar libertad al niño significa, posibilitar los ambientes necesarios para que él pueda desarrollarse, crecer, buscar, indagar, conocer, es decir, investigar. Pero para ello es necesario que las instituciones y los profesores brinden esas posibilidades a través de la utilización de metodologías y didácticas apropiadas en donde no se le esté diciendo todo el tiempo, jestá mal!, sino que se le lleve a que encuentre en dónde está el error.

La actividad es un aspecto del nuevo modelo de enseñanza que adquiere categoría de valor no por si misma, sino por la posibilidad que ofrece de realizar una enseñanza integrada que ayude al alumno a comprender y a desenvolverse en su entorno de modo autónomo, libre y con una actitud crítica".

Pero esa actividad debe ser bien comprendida no como actividad por actividad. sino como la posibilidad de llevar al niño a volver sobre aquello que ha hecho, para reflexionar y poder justificar las decisiones que ha tomado, las estrategias de trabajo que diseñó, la posibilidad de mejorar, modificar o cambiar aquello que realizó. Desde este planteamiento la actividad es consciente, pensada y evaluada y llevada al niño a descubrir nuevos aprendizajes, además esos aprendizajes son significativos para él, por la intervención y participación que ha tenido y lo va estructurando para su desarrollo en la vida.

No se cebe olvidar que el tipo de sociedad en que se desenvuelve el individuo es el que indica el modelo de hombre que se quiere formar por lo tanto "Se presenta como objetivo construir una sociedad más gusta y solidaria". 


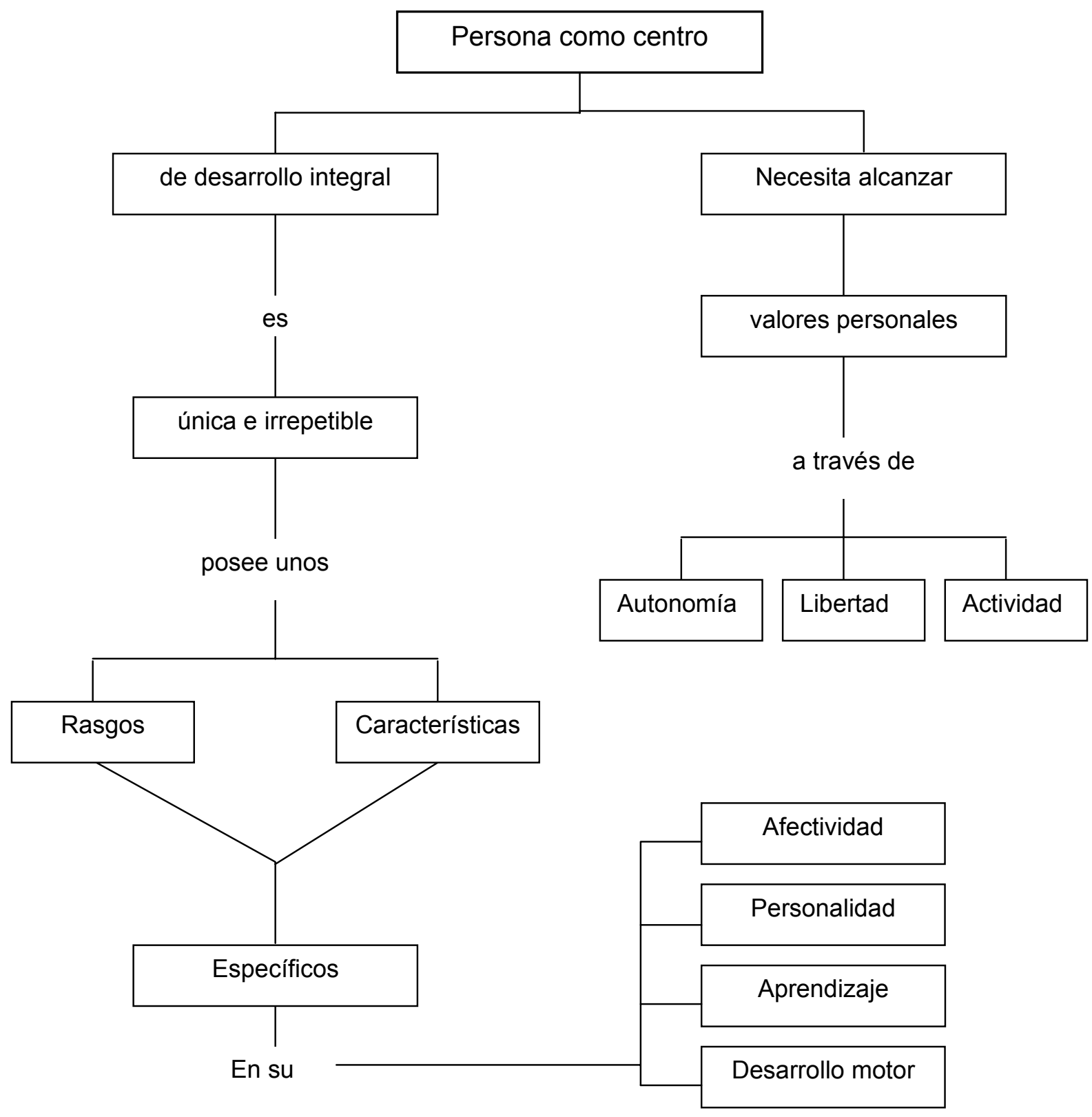

Esto significa que los niños tienen igualdad ce oportunidades para participar en toos los trabajos escolares, se puede relacionar con todos sus compañeros y profesores el intercambio de ideas y tareas le da la posibilidad de reflexionar aclarar sus dudas y ampliar sus conocimiento, es decir lograr su pleno desarrollo

Es necesario tener en cuenta que "Todo valor se desarrolla en un mundo de relaciones; la relación es un principio que subyace a todo sistema social". 
Por lo tanto el niño no está solo en la escuela él es un elemento de interacción, sus ideas, acciones y actividades le permiten compartir, intercambiar y empezar a aceptar el otro, a respetarlo y a tolerarlo. Cuando un profesor le' dice a un niño. "si Juan le pegó, vaya y le devuelve el golpe, está creando resentimientos, odios, venganzas que sólo traerán violencia.

Lo más pertinente en estos casos es utilizar el diálogo y llevar a los niños a que reflexionen sobre las acciones que hicieron y el por qué no las deben hacer. Es necesario llevarlos a valorar sus costumbres, expresiones y creencias, porque éstas son resultado de la apropiación cultural de las comunidades. Pero no todas las costumbres y creencias pertenecen a la formación de un sistema de valores útil al hombre, así por ejemplo: A una comunidad que habita una zona de alta sismicidad, de deslizamientos o cerca de ríos y quebradas que representan un peligro para la vida humana, se le informa sobre los riesgos de su permanencia allí, se le trata de reubicar, pero él se niega a abandonar la región y da una serie de explicaciones como la de considerar que el ama su tierra, que es el lugar donde habitaban sus antepasados. y como en ese sitio nacieron, allí deben morir, sus valores le permite afirmar que forman parte de esa tierra y es imposible abandonarla

Estas situaciones son las que se le deben clarificar a los niños, porque lo más importante para el hombre es la vida, por lo tanto deben tratar de conservarla. El primer valor es el derecho y el deber de conservar la vida. El mantener creencias y costumbres que generan contravalor es un punto de clarificación en la prevención de desastres.

Así mismo algunas comunidades viven del trabajo de cortar los árboles, de negociar con su venta, de sacar leña para cocer los alimentos: es decir, de ahí se deriva su sustento; la no clarificación del valor: protección, saneamiento y recuperación ambiental es lo que ha llevado a la erosión de grandes extensiones de terrenos.

Por lo tanto los niños, deben aprender a reflexionar sobre los valores ambientales. Con el paso de los años, en las veredas, municipios y ciudades, si están siendo deteriorados, por el mal manejo y uso inadecuado que el hombre está haciendo de sus recursos: y por la falta de valores colectivos y autoestima, las montañas, laderas, mesetas y campos están siendo destruidos, las aguas contaminadas y la atmósfera recibe elementos químicos inapropiados para la vida del hombre. Podemos afirmar que la falta de ética ambiental y participación comunitaria son las que ejercen de contravalor y dominan en muchas de nuestras comunidades

\section{El sentido de pertenencia}

Es un valor óptimo y flexible que debe permitir el análisis lógico del espacio donde se habita, lo que lo lleva a un reconocimiento de las fortalezas y debilidades del entorno. Los valores en prevención de desastres van ligados al sentido de pertenencia y se conciben como interacciones entre el valor responsabilidad, respeto activo y autoestima y sus respectivos macrovalores y contravalores. Ver figura 2.

La responsabilidad es individual y colectiva; la responsabilidad individual es la capacidad que tiene una persona de conocer y aceptar las consecuencias de sus actos libres y conscientes.

La responsabilidad colectiva es la capacidad de influir en lo posible en las decisiones de una colectividad, al mismo tiempo que respondemos de las decisiones que se toman como grupo social en donde estamos incluidos. Las interacciones entre algunos valores 
respecto a la responsabilidad se explican en la figura 2. Es de anotar que la responsabilidad como macrovalor conlleva a formar el sentido de pertenencia en tanto que, como contravalor causa un alto sentimiento de injusticia y resentimiento social que afecta la vida del hombre.
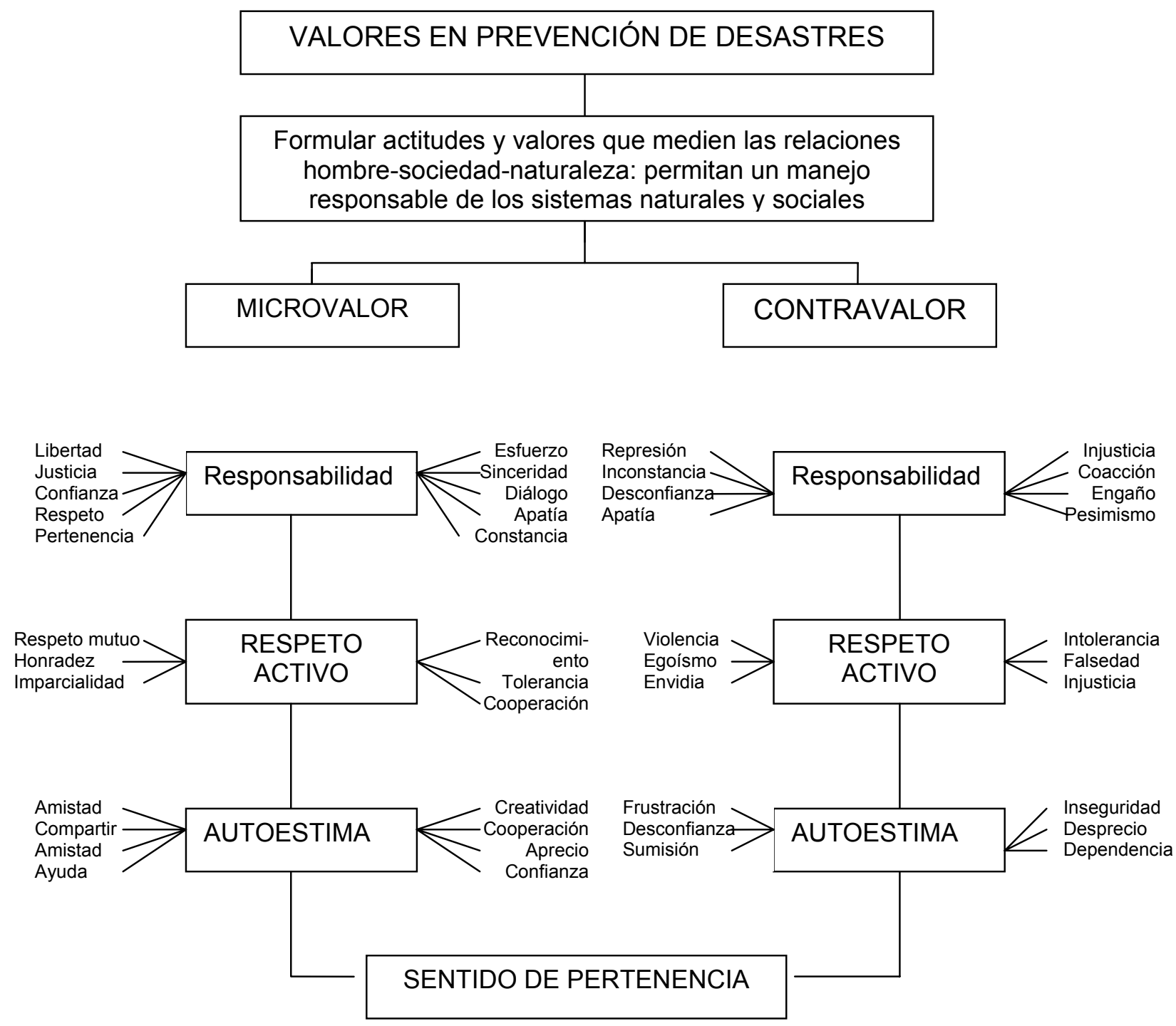

Figura 2

El respeto activo es un macrovalor indispensable en la convivencia y a él se integran la tolerancia, el reconocimiento, la cooperación y la imparcialidad, los cuales son valores esenciales de la minimización del riesgo y la vulnerabilidad hacia el desastre.

La autoestima es la percepción que tiene un individuo sobre sus propios méritos actitudes, o dicho de otro modo, es el concepto de nuestra valía personal y de nuestra capacidad. Esta se construye a partir de las personas que nos rodean, de las experiencias, vivencias y sentimientos que se producen durante todas las etapas de la 
vida; de aquí el aspecto variable de este valor. Las etapas más importantes para su adquisición son la infancia y la adolescencia.

La visión que tiene la persona de si misma, viene determinada por la valoración que han hecho las personas más importantes de su vida, ya sean padres o educadores La persona con autoestima alta es aquella que acepta sus características físicas y psicológicas y es capaz de afrontar cualquier reto que se le presente, ante la situación riesgo de desastre.

\section{EL PROGRAMA DE EDUCACION AMBIENTAL}

El programa de educación ambiental que incorpora la dimensión prevención de desastres al currículo de $\mathrm{V}$ grado de educación básica, tiene como fin primordial la formación de valores humanos, mediante la construcción de un proyecto de educación ambiental en prevención de desastres para conservar las montañas de la cordillera oriental.

Los materiales pedagógicos que apoyan el programa son el módulo de docentes y los cinco módulos escolares sobre los temas de la montaña, el ecosistema humano, los seres vivos, el planeta tierra y las máquinas. Dichos materiales fueron validados en los municipios de Choachi y Útica y con ellos se pretende integrar la ética al programa de Ciencias Naturales.

Los macrovalores redundan en beneficio de la formación de un individuo autónomo, seguro de su razonamiento participativo y autogestionario, capaz de intervenir activamente en el desarrollo de su comunidad y la prevención de desastres, esto lleva a la construcción de una ética, que es el resultado de las interacciones entre los individuos y la colectividad dentro de una dinámica sociedad-naturaleza. Estas interacciones permiten que el individuo analice su realidad, y sea capaz de argumentar y buscar el consenso, basado en el respeto a la diferencia para una toma consciente de decisiones. Se plantea como una nueva educación basada en la responsabilidad, en la crítica, en la adquisición de estructuras mentales, que lo lleven a comprender para actuar.

Para que el niño tome conciencia del cuidado, prevención y protección del ambiente, es importante que pueda comprobar los cambios que se están dando a través de la percepción que haga de su entorno. Es necesario que observe y registre los cambios de temperatura, el aumento o escasez de las precipitaciones, los cambios que se dan en las plantas, el proceso que siguen para tomar los nutrientes del suelo; de esta forma es posible que los niños detecten, analicen y determinen los cambios ambientales que se están dando, e identifiquen las situaciones de riesgo, y las amenazas a que están expuestos, hasta lograr internalizar valores ambientales y valores humanos

\section{BIBLIOGRAFÍA}

1 CAÑÓN, C. El sentido de lo humano. Universidad Javeriana. Bogotá, 1990.

2. CARRERAS, L. et al. Cómo educar. Editorial Narcea Madrid, 1997.

3. DOMINGUEZ. G. Los valores en la educación infantil. Editorial La Muralla. España. 1996. 
4. HARTMAN. Estudios fenomenológicos. E Paidós. Buenos Aires, 1970.

5. MANRIQUE, Max. "Los valores, expresión de armonía social, en: Aula Urbana, 5 Mayoabril, 1998.

6. MARTÍNEZ, J. La formación en valores. Editorial Bruño. Madrid, 1994.

7. NOVACK. J. Aprender a aprender Editorial Iberia. Madrid, 1992.

8. SURDA, J. COLOM, A. Pedagogía ambiental. CE AC, Barcelona, 1989.

9. TALERO, E.; UMAÑA, O. El proyecto de educación ambiental en prevención de desastres. Universidad Pedagógica Nacional. 1998.

10. WILCHES, O. Un programa de prevención de desastres. U. Popayán, 1988, 